\title{
Prevalence and prognosis of non-ischemic patterns of late gadolinium enhancement in older adults by cardiovascular MR in the ICELAND-MI study
}

\author{
Sujata M Shanbhag ${ }^{7 *}$, Thor Aspelund ${ }^{1,2}$, Anders M Greve ${ }^{3,7}$, Gudmundur Thorgeirsson ${ }^{4}$, Erik B Schelbert ${ }^{5,7}$, \\ Jie J Cao ${ }^{6,7}$, Sigurdur Sigurdsson ${ }^{1}$, Peter Kellman7, Gudny Eiriksdottir ${ }^{1}$, Tamara Harris ${ }^{8}$, Lenore Launer ${ }^{8}$, \\ Vilmundur Gudnason', Andrew E Arai ${ }^{7}$
}

From 19th Annual SCMR Scientific Sessions

Los Angeles, CA, USA. 27-30 January 2016

\section{Background}

Late gadolinium enhancement (LGE) can detect and discriminate myocardial scar/fibrosis of ischemic and non-ischemic etiologies. Our objective was to determine the prevalence and prognosis for ischemic and nonischemic patterns of LGE in a community-based sample of older adults.

\section{Methods}

ICELAND-MI is a nested cohort of the Age, Gene/ Environment Susceptibility-Reykjavik Study of community-dwelling older adults that intentionally oversampled diabetic subjects. After excluding subjects with pre-existing heart failure, the cohort size was 900. CMR was used to detect myocardial infarction (MI), major patterns of non-ischemic patterns of LGE as defined by Vöhringer (Herz 2007;32:129-37), and minor patterns of non-ischemic LGE including LGE near the aortic root, mitral annulus, or right ventricular insertion points. The composite end-point was adjudicated hospitalization for heart failure and death.

\section{Results}

The median age was 76 (IQR 72-81), 48\% were male, and $35 \%$ had diabetes. The prevalence of MI, major non-ischemic patterns of LGE, and minor non-ischemic patterns of LGE were $23.4 \%(\mathrm{~N}=211), 6.0 \%(\mathrm{~N}=54)$, and $26.4 \%(\mathrm{~N}=238)$ respectively. Major non-ischemic
LGE demonstrated the highest risk (HR 3.5, p $<0.0001$, Figure 1), MI had similar risk (HR 2.5, $\mathrm{p}<0.0001$ ), and minor non-ischemic LGE had lower but significantly higher risk (HR1.5, $\mathrm{p}=0.03$ ) compared to those without LGE. Controlling for age, gender, LVEF, diabetes, and hypertension, major non-ischemic LGE remained strongly predictive of the composite endpoint (HR 2.3, $\mathrm{p}=0.001$ ) while minor non-ischemic patterns of LGE were of borderline significance $(\mathrm{p}=0.07)$.

\section{Conclusions}

Subjects with major non-ischemic LGE patterns are at increased risk of developing heart failure and death.

\section{Authors' details}

${ }^{1}$ Icelandic Heart Association, Kopavogur, Iceland. ${ }^{2}$ Public Health Sciences, University of Iceland, Reykjavik, Iceland. ${ }^{3}$ Gentofte Hospital, Copenhagen, Iceland. ${ }^{4}$ University of Iceland, Reykjavik, Iceland. ${ }^{5}$ Division of Cardiology, UPMC Heart and Vascular Institute, Pittsburgh, PA, USA. ${ }^{6}$ Cardiology, St. Francis Hospital, Roslyn, NY, USA. ${ }^{7} \mathrm{NIH} / \mathrm{NHLB}$, Bethesda, MD, USA. ${ }^{8}$ National Institute on Aging, NIH, Bethesda, MD, USA.

Published: 27 January 2016

doi:10.1186/1532-429X-18-S1-061

Cite this article as: Shanbhag et al:: Prevalence and prognosis of nonischemic patterns of late gadolinium enhancement in older adults by cardiovascular MR in the ICELAND-MI study. Journal of Cardiovascular Magnetic Resonance 2016 18(Suppl 1):O61. 


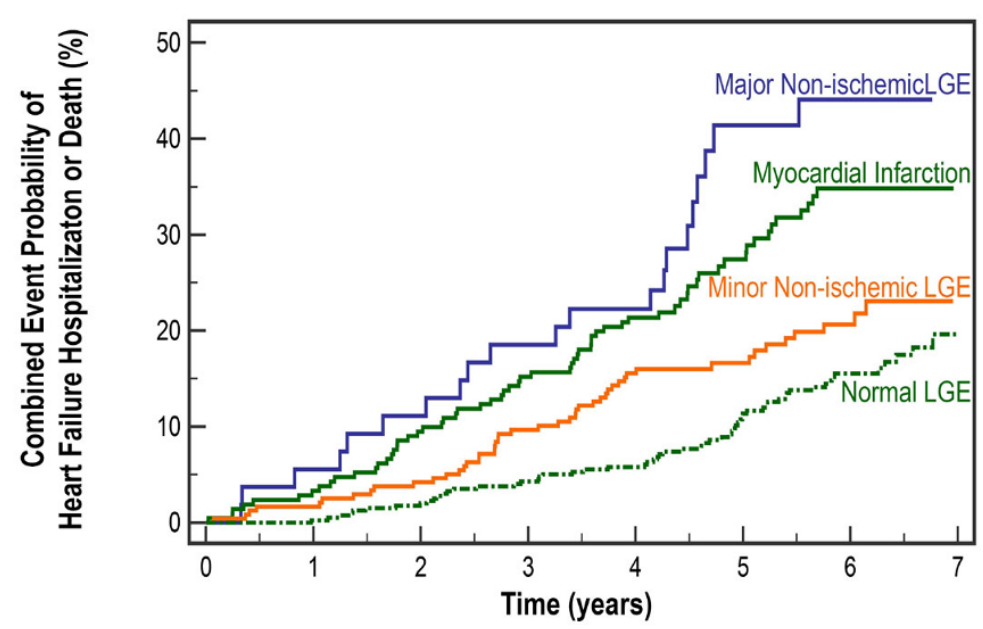

\section{Number at Risk}

$\begin{array}{lllllllll}\text { Major Non-ischemic LGE } & 54 & 51 & 48 & 44 & 41 & 22 & 16 & 0\end{array}$

$\begin{array}{lllllllll}\text { Myocardial Infarction } & 211 & 204 & 191 & 179 & 165 & 100 & 65 & 0\end{array}$

Minor Non-ischemic LGE $238 \quad 234 \quad 228 \quad 215 \quad 201 \quad 129 \quad 71 \quad 0$

$\begin{array}{lllllllll}\text { Normal LGE } & 397 & 396 & 389 & 380 & 370 & 290 & 200 & 0\end{array}$

Figure 1 Kaplan-Meier Event Rates for Subjects with Major Non-ischemic LGE, MI, Minor Non-ischemic LGE versus Normal LGE. 